\title{
Analysis of Direct Numerical Simulations of ignition fronts in turbulent non-premixed flames in the context of Conditional Moment Closure
}

\author{
E.S. Richardson ${ }^{1}$, N. Chakraborty ${ }^{2}$ AND E. Mastorakos ${ }^{1}$ \\ 1: Hopkinson Laboratory, Department of Engineering, \\ University of Cambridge, CB2 1PZ, U.K. \\ 2: Department of Engineering, University of Liverpool, \\ Brownlow Hill, L69 3GH, U.K.
}

Corresponding author:

E.S. Richardson

Hopkinson Laboratory, Department of Engineering,

University of Cambridge, Trumpington Street,

Cambridge, CB2 1PZ, U.K.

Tel.: +44 (0)1223 332690

Fax: +44 (0)1223 332662

E-mail: esr24@cam.ac.uk

Colloquium Topic: Turbulent Flames, Non-premixed

Short Running Title: Conditional turbulent flux in ignition kernels

Word Count:

Text: 3174

Figures: 1: 123; 2: 125; 3: 236; 4: 470; 5: 468; 6: 473

Equations: 175

References: 420

Tables : 0 words

Total: 5784 words

Total: 7.3 pages

Accepted for oral presentation in

$31^{\text {st }}$ International Symposium on Combustion 


\begin{abstract}
Direct Numerical Simulations of an igniting non-premixed flame are analysed in order to assess the accuracy of the gradient diffusion model for the conditional turbulent flux term in the CMC equation. This term may become important in realistic combustion situations involving stabilisation, ignition and extinction. The results show that the edge flame probably cannot be treated with first-order CMC and that its structure involves a balance between chemistry, molecular mixing, and spatial transport. The usual gradient diffusion approximation for the conditional turbulent flux seems to be adequate for high turbulence intensity relative to the laminar burning velocity of a stoichiometric mixture, but there is strong counter-gradient transport at weak turbulence. An analysis of the major terms in the conditional scalar flux equation shows that the pressure fluctuation is mainly responsible for the counter-gradient transport.
\end{abstract}

\title{
Keywords
}

Forced ignition, non-premixed, Conditional Moment Closure. 


\section{Introduction}

The Conditional Moment Closure is one of the most advanced turbulent combustion models [1]. It aims to solve transport equations for the conditionally-averaged mass fractions and temperature defined as $Q_{\alpha}(\eta, x, t)=\left\langle\rho Y_{\alpha}(x, t) \mid \xi(x, t)=\eta\right\rangle / \rho_{\eta}$ and $Q_{T}(\eta, x, t)=\langle\rho T(x, t) \mid \xi(x, t)=\eta\rangle / \rho_{\eta}$ where $\alpha$ denotes a species, $T$ the temperature, $\xi$ is the mixture fraction, $\eta$ is the sample space variable for the conserved scalar, $\langle\cdot \mid \xi=\eta\rangle$ denotes ensemble averaging subject to the condition that $\xi=\eta$; in short $Q_{\alpha}=\left\langle Y_{\alpha} \mid \eta\right\rangle \cdot \rho_{\eta}$ is the conditionally-averaged density $\langle\rho \mid \xi=\eta\rangle$. The symbol $\langle\cdot\rangle$ denotes in this paper unconditional average. A double-prime denotes a Favre fluctuation about the conditional average $Q$, while a single prime an unconditional fluctuation. The governing equation for the conditional average species mass fraction is [1]

$$
\frac{\partial Q_{\alpha}}{\partial t}=-\left\langle u_{i} \mid \eta\right\rangle \frac{\partial Q_{\alpha}}{\partial x_{i}}+\langle N \mid \eta\rangle \frac{\partial^{2} Q_{\alpha}}{\partial \eta^{2}}+\rho_{\eta}\left\langle\dot{w}_{\alpha} \mid \eta\right\rangle-\frac{1}{\bar{\rho} \widetilde{P}(\eta)} \frac{\partial}{\partial x_{i}}\left(\left\langle u_{i}^{\prime \prime} Y_{\alpha}^{\prime \prime} \mid \eta\right\rangle \bar{\rho} \widetilde{P}(\eta)\right)
$$

$\widetilde{P}(\eta)$ is the density-weighted probability density function of $\xi, \bar{\rho}$ is the mean density, $\dot{w}$ is the reaction rate, and $N$ is (half) the scalar dissipation. A similar equation is available for the enthalpy (or temperature) with added terms due to radiation and pressure work [2]. Equation (1) is a 5dimensional partial differential equation and therefore its solution is a formidable task. However, often the spatial dependence of $Q$ is weak or the flow allows a volume integration to be performed to reduce the dimensionality of the problem [1]. Hence, CMC has been used extensively for nonpremixed jet flames $[3,4]$ with a cross-stream average formulation, resulting in a parabolic equation where the last term in the r.h.s. of Eq. (1) is absent.

$\mathrm{CMC}$ is currently being used for problems of increasing complexity. In many technological applications, it is common to encounter flames that involve significant variations of the conditional averages, for example in regions of ignition and stabilization, and this requires accurate models for the conditional mean velocity and turbulent flux. The latter term is usually modelled as [1]

$$
\left\langle u_{i}^{\prime \prime} Y^{\prime \prime} \mid \eta\right\rangle=-D_{t} \frac{\partial Q}{\partial x_{i}}
$$

where $D_{t}$ is an eddy diffusivity. Lifted flames [5,6], autoignition of gas jets and sprays $[2,7,8]$, bluff-body non-premixed flames [9], and fires [10] have been examined using the model of Eq. (2). 
In some of these, it has been found that the turbulent flux term is important for the overall success of the prediction. For example, a balance of terms in the CMC equation in the stabilisation region of a lifted flame $[5,6]$ shows that this term contributes significantly and hence it was concluded that it is important to examine its modelling. A similar conclusion was reached after exploring the results from two-dimensional solutions of Eq. (1) in an autoigniting spray [2], which showed that CMC could possibly be used for all the phases of diesel engine combustion (autoignition, premixed, non-premixed), as long as the spatial transport terms are captured correctly.

Motivated by these trends and suggestions concerning these CMC sub-models, in this paper, we examine Direct Numerical Simulations of a turbulent non-premixed flame with a propagating edge, a situation of practical interest in terms of spark-ignition of gas turbine flames and of autoignition in diesel engines. The simulations are designed to produce spatial gradients of the conditional averages and the results are analyzed in terms of Eq. (1) and aim, in particular, to examine the validity of Eq. (2). The transport equation for the conditional turbulent flux is also used to assist in the interpretation of the results.

\section{Formulation}

\subsection{Direct Numerical Simulations}

An inert mixing layer of partially premixed fuel and air is allowed to interact with homogeneous isotropic decaying turbulence at time $t=0$. The simulations have been carried out using a threedimensional fully compressible DNS code, SENGA[11, 12]. A single-step irreversible reaction is considered with an Arrhenius expression and the stoichiometry of methane $\left(\xi_{s t}=0.055\right)$. The conservation equations of mass, momentum, energy and mass fractions of fuel and oxidiser are solved in dimensionless form. The specific heats for all species are assumed equal and independent of temperature, Fick's law was used, and the Lewis numbers of all the species were unity. All first and second order derivatives are evaluated using a $10^{\text {th }}$-order central difference scheme, with the order of differentiation gradually dropping to second order one-sided finite difference near the nonperiodic boundaries. The boundaries in the direction of inhomogeneity ( $x$-direction) are taken to be partially non-reflecting using the NSCBC technique. The transverse directions $(y$ and $z)$ are taken 
to be periodic. Time advancement is carried out using a third order Runge-Kutta scheme. The initial turbulent velocity field is generated according to the Batchelor-Townsend spectrum and gives a characteristic velocity scale of $u^{\prime}$ and a lengthscale $L_{t}$. For all simulations, the grid spacing was smaller than the Kolmogorov length scale and about 10 grid points were placed within the thermal thickness of a stoichiometric unstrained laminar premixed flame $\delta=\left(T_{a d}-T_{u}\right) / \max |\nabla \hat{T}|$, where $T_{a d}$ is the adiabatic flame temperature of the stiochiometric mixture, $T_{u}$ is the unburned gas temperature, and $\hat{T}$ is the dimensional temperature. The domain is a cube of length $L\left(L / L_{t} \approx 6\right)$ and all the simulations have been carried out on a grid of $128^{3}$ points with equal spacing in all directions. The non-dimensional temperature $T$ used here is given by $\left(\hat{T}-T_{u}\right) /\left(T_{a d}-T_{u}\right)$. All lengths are non-dimensionalised by $L$ and velocities by the laminar burning velocities of the stoichiometric mixture $S_{L}[11,12]$.

The spark was modelled as a thin cylinder spanning the domain in the $z$ direction in order to form averages over the $\mathrm{z}$ direction. A power source in the energy equation lasting $t_{s p}$ was used, with $t_{s p}=0.2 \alpha / S_{L}^{2}$ to ensure ignition, where $\alpha$ is the thermal diffusivity. The source term followed a Gaussian distribution around the spark centre with an 1/e thickness of $1.5 \delta$. For the spark duration, energy, and size used here, it was possible to have successful self-sustained combustion in quiescent homogeneous stoichiometric fuel-air mixture. Spark ignition implementation is discussed in detail in Ref. [13]. The initial mixture fraction distribution in the inhomogeneous direction is described by an error function and the fuel and oxidiser mass fractions follow inert mixing given by $Y_{F}=Y_{F \infty} \xi, Y_{O}=Y_{O \infty}(1-\xi)$ where the fuel mass fraction in pure fuel $Y_{F \infty} \equiv 1$ and the oxygen mass fraction in pure air $Y_{O \infty} \equiv 0.233$. To improve resolution across the flame zone, the maximum mixture fraction was 0.1 . The spark centre was at $y=0.5$ and at the $x$ where $\xi=\xi_{s t}$. Two simulations were run for weak $\left(u^{\prime} / S_{L}=4\right)$ and strong turbulence $\left(u^{\prime} / S_{L}=12\right)$ lasting at least 1.5 $L_{t} / u^{\prime}$. For both simulations $L_{t} S_{L} / \alpha=3.4$, and the flame Mach number $M a=S_{L} / a_{0}=0.014159$ where $a_{0}$ is the accoustic speed at the reference conditions. In the present study $\rho_{u} / \rho_{b}=4$ for the stoichiometric mixture, where $\rho_{b}$ and $\rho_{u}$ are the burned and unburned gas densities respectively. 


\subsection{CMC equations}

A transport equation for the conditional turbulent flux for a reacting scalar $Y$ can be derived from the decomposition method and the derivation confirmed from the PDF method [1]. Starting from the transport equations for $u_{i}$ and $Y$, equations for $\rho_{\eta}\left\langle u_{i} \mid \eta\right\rangle, \rho_{\eta}\langle Y \mid \eta\rangle, \rho_{\eta}\left\langle u_{i} Y \mid \eta\right\rangle$, and $\rho_{\eta}\left\langle u_{i} \mid \eta\right\rangle\langle Y \mid \eta\rangle$ are derived. These are then used to give the transport equation for $\rho_{\eta}\left\langle u_{i}^{\prime \prime} Y^{\prime \prime} \mid \eta\right\rangle$. The resulting equation becomes:

$$
\begin{array}{r}
\frac{\partial}{\partial t}\left[\rho_{\eta}\left\langle u_{i}{ }^{\prime \prime} Y^{\prime \prime} \mid \eta\right\rangle\right]+\frac{\partial}{\partial x_{j}}\left[\rho_{\eta}\left\langle u_{j} \mid \eta\right\rangle\left\langle u_{i}{ }^{\prime \prime} Y^{\prime \prime} \mid \eta\right\rangle\right]=-\frac{\partial}{\partial x_{j}}\left[\rho_{\eta}\left\langle u_{i}{ }^{\prime \prime} u_{j}{ }^{\prime \prime} Y^{\prime \prime} \mid \eta\right\rangle\right] \\
-\rho_{\eta}\left\langle u_{j}{ }^{\prime \prime} Y^{\prime \prime} \mid \eta\right\rangle \frac{\partial\left\langle u_{i} \mid \eta\right\rangle}{\partial x_{j}} \underbrace{-\rho_{\eta}\left\langle u_{i}{ }^{\prime \prime} u_{j}{ }^{\prime \prime} \mid \eta\right\rangle \frac{\partial\langle Y \mid \eta\rangle}{\partial x_{j}}}_{\text {scalar gradient }} \\
\underbrace{-\left\langle Y^{\prime \prime} \mid \eta\right\rangle \frac{\partial\langle p \mid \eta\rangle}{\partial x_{i}}-\left\langle\frac{\partial p^{\prime \prime}}{\partial x_{i}} Y^{\prime \prime} \mid \eta\right\rangle-\frac{\partial\langle p \mid \eta\rangle}{\partial \eta}\left\langle\frac{\partial \xi}{\partial x_{i}} Y^{\prime \prime} \mid \eta\right\rangle}_{\text {pressure }}+\left\langle Y^{\prime \prime} \mid \eta\right\rangle\left\langle\frac{\partial\left(\mu s_{i j}^{\prime \prime}\right)}{\partial x_{j}} \mid \eta\right\rangle+\left\langle u_{i}{ }^{\prime \prime} \mid \eta\right\rangle\left\langle\frac{\partial}{\partial x_{j}}\left(\rho D \frac{\partial Y^{\prime \prime}}{\partial x_{j}}\right) \mid \eta\right\rangle \\
+\underbrace{\rho_{\eta}\left\langle\dot{w}^{\prime \prime} u_{i}{ }^{\prime \prime} \mid \eta\right\rangle}_{\text {reaction }}+\frac{\partial\left\langle u_{i} \mid \eta\right\rangle}{\partial \eta}\left\langle\frac{\partial}{\partial x_{j}}\left[(\mu-\rho D) \frac{\partial \xi}{\partial x_{j}}\right] Y^{\prime \prime} \mid \eta\right\rangle+\left\langle\rho N Y^{\prime \prime} \mid \eta\right\rangle \frac{\partial^{2}\left\langle u_{i} \mid \eta\right\rangle}{\partial \eta^{2}}+\left\langle\rho S c N u_{i}{ }^{\prime \prime} \mid \eta\right\rangle \frac{\partial^{2}\langle Y \mid \eta\rangle}{\partial \eta^{2}}
\end{array}
$$

with $s_{i j}^{\prime \prime}=\left(\partial u_{i}^{\prime \prime} / \partial x_{j}+\partial u_{j}^{\prime \prime} / \partial x_{i}\right)$ and $S c=\mu /(\rho D)$. There are two additional terms that have been neglected under a high Reynolds number assumption given by $e_{1}=\left\langle\mu \frac{\partial \xi}{\partial x_{j}} \cdot \frac{\partial}{\partial x_{j}}\left(\frac{\partial\left\langle u_{i} \mid \eta\right\rangle}{\partial \eta}\right) Y^{\prime \prime} \mid \eta\right\rangle$ and $e_{2}=\left\langle\rho D \frac{\partial \xi}{\partial x_{j}} \cdot \frac{\partial}{\partial x_{j}}\left(\frac{\partial\langle Y \mid \eta\rangle}{\partial \eta}\right) u_{i}{ }^{\prime \prime} \mid \eta\right\rangle$. The physical interpretation of the various terms in Eq. (3) is as follows. The first and second terms on the l.h.s. signify unsteady accumulation and advection. The first term in the r.h.s. denotes turbulent transport of $\left\langle u_{i}^{\prime \prime} Y^{\prime \prime} \mid \eta\right\rangle$ and the following two terms signify transport of conditional flux due to conditional mean velocity and scalar gradients. The remainder of the r.h.s. contains contributions due to the mean and fluctuating pressures, molecular diffusion, chemistry, and the correlations between the velocity and scalar and the scalar dissipation $N$. It is instructive to compare Eq. (3) with the more familiar unconditional scalar flux $\left\langle\rho u_{i}^{\prime} Y^{\prime}\right\rangle$ transport equation (e.g. $[14,15])$. That equation contains terms of similar nature and structure, but lacks the $\eta$-space derivatives that are present in Eq. (3). The magnitude of the various terms in the conditional scalar flux equation has been measured from the DNS data and is presented later. 


\section{Results and Discussion}

\subsection{Flow patterns}

Assuming that the mixture fraction obeys a gradient diffusion process, an effective diffusivity $D_{t}$ has been calculated from the evolution of the $\langle\rho \xi\rangle$ profile in the $x$-direction (averaging being performed over $y-z$ planes) by measuring $\left\langle\rho v^{\prime} \xi^{\prime}\right\rangle$ and dividing by $-\partial\langle\rho \xi\rangle / \partial x$. This always gave a positive number that changed very little in time and space. In a calculation, this eddy diffusivity would be used in Eq. (2) and hence we can now test the gradient diffusion model. Some details on the flame patterns examined are given next.

Figure 1 shows that the thin spark cylinder creates a broadly cylindrical flame that expands in the $x$ and $y$ directions and gets distorted by the turbulence. High temperature zones at fuel rich $\left(\xi>\xi_{s t}\right)$ and lean $\left(\xi<\xi_{s t}\right)$ locations in Fig. 1b correspond to rich and lean premixed flames respectively whereas the high temperature zone on the $\xi_{s t}$ isosurface originates due to a diffusion flame. More details on flame structure can be found elsewhere [13]. The flame expands in the $y$-direction along the $\xi_{s t}$ iso-surface, while it expands in the $x$-direction across mixture fraction contours.

Figure $2 \mathrm{a}$ and $2 \mathrm{~b}$ show the measured unconditional turbulent flux and the one estimated from the gradient diffusion hypothesis. The data are for $u^{\prime} / S_{L}=4$. It is clear that some evidence of counter-gradient diffusion is visible at a few locations. This is not the case for $u^{\prime} / S_{L}=12$, where $\left\langle\rho v^{\prime} T^{\prime}\right\rangle$ and $-D_{t} \partial\langle\rho T\rangle / \partial y$ always have the same sign (not shown). The fact that counter-gradient diffusion is evident at weak turbulence but not at high $u^{\prime} / S_{L}$ has been thoroughly analyzed in the context of premixed flames (e.g. [16]) and the present data suggest that the same mechanisms may also apply in the context of partially-premixed and edge flames.

\subsection{CMC equation analysis}

In this Section, we present conditional averages and the balance of terms in the CMC equation. To improve statistical convergence, the following procedure has been used. First, the conditional averages of a scalar $\phi$ using data along a $z$ line are used to get a first estimate of the conditional average, $\langle\phi \mid \eta\rangle_{1}(x, y)$, which is a function of $x$ and $y$. Usually, there isn't enough data to populate all $\eta$ space. Then, a cross-stream average is calculated by [1] 


$$
\langle\phi \mid \eta\rangle(y)=\frac{\int\langle\phi \mid \eta\rangle_{1}(x, y) \widetilde{P}(\eta, x, y) d x}{\int \widetilde{P}(\eta, x, y) d x}
$$

The resulting cross-stream averaged CMC equation, for example for the conditional mass fraction, has a dependence in space only in the $y$-direction:

$$
\frac{\partial Q}{\partial t}=-\langle v \mid \eta\rangle \frac{\partial Q}{\partial y}+\langle N \mid \eta\rangle \frac{\partial^{2} Q}{\partial \eta^{2}}+\rho_{\eta}\langle\dot{w} \mid \eta\rangle-\frac{1}{\bar{\rho} \widetilde{P}(\eta)} \frac{\partial}{\partial y}\left(\left\langle v^{\prime \prime} Y^{\prime \prime} \mid \eta\right\rangle \bar{\rho} \widetilde{P}(\eta)\right)
$$

The conditionally-averaged temperature is shown in Fig. 3a. It is evident that the thickness of the flame brush in the $y$-direction (i.e. the main direction of propagation of the edge flame) is not small (of the order of $L_{t}$ ) and that it increases with time from the spark. It is also evident that the flame edge at the high turbulence case propagates slower than the flame at the lower $u^{\prime} / S_{L}$. This is consistent with the finding from laminar triple flames that high strain rates decrease the flame propagation speed and can also make it negative relative to the local flow speed (i.e. a retreating front) $[13,17]$. The pressure increases at the centre of the spark as a result of the sudden increase in temperature. The pressure decreases from the hot region in the centre of the domain, with the pressure gradient becoming smaller with time (Fig. 3b). The pressure gradient in $\eta$ space in the present case is found to be negligible compared to the pressure gradient in physical space.

The situation studied here is statistically unsteady and hence the sum of terms in the r.h.s. of Eq. (5) is not zero. Figure 4 shows the distribution of these terms in the $y$-direction for $\eta=\xi_{s t}$ for the fuel mass fraction. The lack of complete symmetry around $y=0.5$ is due to the limited sample size. The role of the turbulent flux term differs between the low and high turbulence cases. For $u^{\prime} / S_{L}=4$ the turbulent flux acts to move fuel from the burnt side of the flame to the unburned side, against its mean gradient. In the higher turbulence case, Figs. 5b,d, the reverse is true and the turbulent flux is seen to increase the fuel concentration within the burned region. In all cases the molecular mixing counteracts the chemical consumption of fuel. Its magnitude, relative to the chemistry term, increases with the turbulence level and with time. In the high turbulence case, the profile of the reaction, molecular mixing, and turbulent flux terms through the edge flame is qualitatively the same as that found in the leading edge of a lifted jet flame [6]. Since the only mean flow is that induced by the expansion of the kernel, the convection term is relatively weak 
and it is noted that it opposes the turbulent flux term in every case.

It is interesting to assess the accuracy of the first-order CMC model for this problem because most elliptic CMC calculations have been performed to this level. Figure 4 also contains the distribution of the reaction rate evaluated at the mean conditional species mass fractions and

temperature. It is evident that the first-order closure gives a large error in the reaction rate compared to $\rho_{\eta}\langle\dot{w} \mid \eta\rangle$, particularly in the flame fronts where the conditional temperature fluctuations are high, with the normalized variance $\left\langle T^{\prime \prime 2} \mid \eta=\xi_{s t}\right\rangle /\left(T_{a d}\left(1-T_{a d}\right)\right) \approx 0.6$ (not shown here). This discrepancy suggests that second-order closure or double-conditioning may be necessary for this problem [18-20].

It is evident that a complex spatial structure emerges in turbulent non-premixed edge flame propagation. In order to successfully capture these phenomena, the conditional velocity and conditional flux terms must be adequately modelled, in addition to the mean reaction rate. Various models for $\left\langle u_{i} \mid \eta\right\rangle$ have already been tested [21] and hence we focus here on an evaluation of the usual conditional turbulent flux model of Eq. (2).

\subsection{Conditional fluxes}

Figure 5a,b shows a comparison between $\left\langle v^{\prime \prime} T^{\prime \prime} \mid \eta\right\rangle$ and the gradient model $-D_{t} d\langle T \mid \eta\rangle / d y$ for $\eta=\xi_{s t}$ as a function of $y$, and Figs. 5c,d show the same terms in $y=0.5$ as a function of $\eta$. It is evident that the low turbulence case shows evidence of counter-gradient diffusion at early times and at some $\eta$. In contrast, the gradient diffusion model is adequate for the high turbulence case for all mixture fractions. We conclude that the usual model in Eq. (2) is adequate for high turbulence flames. Note that the present observation that $u^{\prime} / S_{L}=4$ shows counter-gradient diffusion, but $u^{\prime} / S_{L}=12$ does not, is quantitatively consistent with the premixed flame data of Ref. [16] that give the same behaviour at $u^{\prime} / S_{L}=3.6$, but a gradient flux at $u^{\prime} / S_{L}=8.8$. We may conjecture that the turbulent edge non-premixed flame propagation involves qualitatively similar dependencies of the fluxes to the better-known fully premixed flames.

The major terms in the conditional flux equation, Eq. (3), are shown in Fig. 6 for the temperature. The terms not shown are consistently very small. The terms that remain are the mean and fluctuating pressure terms ( $-\left\langle T^{\prime \prime} \mid \eta\right\rangle \partial\langle p \mid \eta\rangle / \partial y$ and $-\left\langle\left(T^{\prime \prime} \partial p^{\prime \prime} / \partial y\right) \mid \eta\right\rangle$ respectively), the conditional 
temperature gradient $\left(-\left\langle v^{\prime \prime} v^{\prime \prime} \mid \eta\right\rangle \partial\langle T \mid \eta\rangle / \partial y\right)$, and the chemistry-velocity correlation $\left(\left\langle\dot{w}^{\prime \prime} u_{i}^{\prime \prime} \mid \eta\right\rangle\right)$. The way these plots have been made, together with the conditional mean temperature profile in Fig. 3, imply that a negative (positive) contribution in the region $\partial\langle T \mid \eta\rangle / \partial y>0$ and a positive (negative) contribution in $\partial\langle T \mid \eta\rangle / \partial y<0$ suggest gradient (counter-gradient) transport. Only the $y$-dependence in $\eta=\xi_{s t}$ is shown, however the main qualitative features are the same in all $\eta$.

An examination of Fig. 6 shows that, in general, the fluctuating pressure term is usually the largest. In both high and low $u^{\prime} / S_{L}$ cases, the temperature gradient contribution promotes gradient transport. The pressure fluctuation contributions act predominantly in a counter-gradient way in the flame fronts, however it can promote gradient diffusion in the burned gas. In the high turbulence case the mean pressure term acts predominantly in the direction of gradient transport, whereas in the low turbulence case it promotes counter-gradient transport, especially in the reaction fronts. The chemistry term is counter-gradient in the low turbulence case and it is gradient in nature for the high turbulence case, but for both it is important only in the reaction front. Figure 5 shows that the degree of counter-gradient transport in the low turbulence case decreases with time. This is because in these simulations, the spark energy initially increases the temperature above the adiabatic flame temperature, which enhances the effects of heat release and thereby enhances the counter-gradient transport. Indeed Fig. 3 shows that in the simulations where the maximum $\langle T \mid \eta\rangle$ is high, counter-gradient transport is strongly promoted. In general, the reasons for the presence of counter-gradient diffusion in Fig. 5 revealed by Fig. 6 are similar to those in fully premixed flames [14, 22]. In Ref. [14], the parameter $\tau S_{L} / u^{\prime}$ was used qualitatively to distinguish between counter-gradient $\left(\tau S_{L} / u^{\prime} \gg 1\right)$ and gradient $\left(\tau S_{L} / u^{\prime} \ll 1\right)$ transport, where $\tau$ is equal to $\rho_{u} / \rho_{b}-1$. In the low turbulence flame $\tau S_{L} / u^{\prime}$ is nominally 0.75 , but in reality it is higher because the spark energy increases the burning velocity and $u^{\prime}$ decays at the flame front. This parameter is much smaller than unity in the high turbulence case. Hence the present non-premixed edge flame data are qualitatively consistent with the expectations from premixed flames.

We conclude that modelling the propagation of turbulent non-premixed edge flames must involve a more sophisticated model than that in Eq. 2, if the model is to be valid for a wide range of $u^{\prime} / S_{L}$. However, for realistic combustors where $u^{\prime} / S_{L}$ is expected to be high, for example 10 , the current CMC modelling approach may be an acceptable approximation. 


\section{Conclusions}

Direct Numerical Simulations of an igniting non-premixed flame are analysed to assess the accuracy of the gradient diffusion model for the conditional turbulent flux term in the CMC equation. The results show that the first-order model for the conditionally-averaged reaction rate is not accurate at the edge flame, indicating that more advanced closures may be required. The edge flame structure involves a balance between chemistry, molecular mixing, and spatial transport, which implies that modelling the conditional convection and diffusion are important for capturing flame establishment following ignition, e.g. in diesel engines or gas turbines. The usual gradient diffusion approximation for the conditional turbulent flux seems to be adequate for high turbulence intensity relative to the laminar burning velocity of a stoichiometric mixture, but there is strong countergradient diffusion at weak turbulence. An analysis of the major terms in the conditional scalar flux equation shows that the pressure fluctuation is mainly responsible for counter-gradient transport.

\section{Acknowledgments}

This work has been funded by the EPSRC, the Department of Trade and Industry, and RollsRoyce Plc. We thank Dr. R.S. Cant for useful discussions and for the permission to use the code SENGA. 


\section{References}

[1] A.Y. Klimenko and R.W. Bilger. Prog. Energy Combust. Sci., 25:595-687, 1999.

[2] Y. M. Wright, G. de Paola, K. Boulouchos, and E. Mastorakos. Combust. Flame, 143:402-419, 2005 .

[3] M.R. Roomina and R.W. Bilger. Combust. Flame, 125:1176-1175, 2001.

[4] M. Fairweather and R.M. Woolley. Combustion and Flame, 133:393-405, 2003.

[5] C.B. Devaud and K.N.C. Bray. Combust. Flame, 132:102-114, 2003.

[6] I.S. Kim and E. Mastorakos. Proc. Combust. Inst., 31:911-918, 2005.

[7] S.H. Kim, K.Y. Huh, and R.A. Fraser. Proc. Combust. Inst., 28:185-191, 2000.

[8] W.T. Kim and K.Y. Huh. Proc. Combust. Inst., 29:569-576, 2002.

[9] S.H. Kim, K.Y. Huh, and L. Tao. Combust. Flame, 120:75-90, 2000.

[10] M.J. Cleary and J.H. Kent. Combust. Flame, 143:357-368, 2005.

[11] N. Chakraborty. PhD thesis, University of Cambridge, 2004.

[12] N. Chakraborty and R.S. Cant. Phys. Fluids, 17, 2005. Article No. 105105 (1-20).

[13] N. Chakraborty, E. Mastorakos, and R.S. Cant. Combust. Sci. Tech, to appear 2006.

[14] D. Veynante, A. Trouvé, K.N.C. Bray, and T. Mantel. J. Fluid Mech., 332:263-293, 1997.

[15] K.H. Luo. Proc. Combust. Inst., 28:489-495, 2000.

[16] J.H. Frank, P.A.M. Kalt, and R.W. Bilger. Combust. Flame, 116:220-232, 1999.

[17] H.G. Im and J.H. Chen. Combust. Flame, 126:1384-1392, 2001.

[18] S.H. Kim, K.Y. Huh, and R.W. Bilger. Proc. Combust. Inst., 29:2131-2137, 2002.

[19] C.M. Cha and H. Pitsch. Combust. Theory Modelling, 6:425-437, 2002.

[20] A. Kronenburg and M. Kostka. Combust. Flame, 143:342-356, 2005.

[21] N Swaminathan and R.W. Bilger. Combust. Theory Modelling, 5:241-260, 2001.

[22] D. Veynante and T. Poinsot. J. Fluid Mech., 353:83-114, 1997. 


\section{List of Figures}

1 The stoichiometric iso-surface (blue) and the iso-surface of $T=0.5$ (red) for $u^{\prime} / S_{L}=4.14$

2 Contours of unconditional flux in the $y$-direction and of the usual gradient model at $t=2.1 t_{s p}$ and for $u^{\prime} / S_{L}=4 \ldots \ldots \ldots \ldots \ldots \ldots \ldots \ldots \ldots \ldots \ldots \ldots \ldots \ldots \ldots$

3 Distributions in the $y$-direction of the mean conditional temperature and pressure

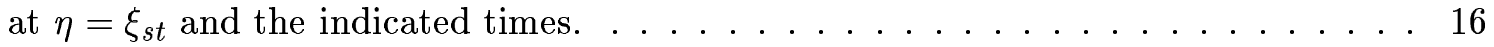

4 The spatial distribution of the various source terms in the CMC equation at $\eta=\xi_{s t}$. (a): $u^{\prime} / S_{L}=4, t=2.1 t_{s p} ;(\mathrm{b}): u^{\prime} / S_{L}=12, t=1.05 t_{s p} ;(\mathrm{c}): u^{\prime} / S_{L}=4, t=4.725 t_{s p}$; $(\mathrm{d}): u^{\prime} / S_{L}=12, t=3.15 t_{s p} \ldots \ldots \ldots \ldots \ldots \ldots \ldots \ldots \ldots \ldots \ldots \ldots \ldots \ldots \ldots$

5 Turbulent flux $(*)$ and gradient diffusion approximation (o) for the indicated $u^{\prime} / S_{L}$ and time. For (a) and (b) the $y$-distribution of $\left\langle v^{\prime \prime} T^{\prime \prime} \mid \eta=\xi_{s t}\right\rangle$ is shown. For (c) and

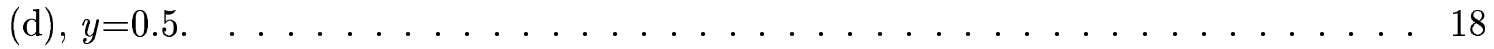

6 The spatial distribution of the various source terms in the CMC conditional flux equation for $\eta=\xi_{s t}$. (a): $u^{\prime} / S_{L}=4, t=2.1 t_{s p}$; (b): $u^{\prime} / S_{L}=12, t=1.05 t_{s p}$; (c): $u^{\prime} / S_{L}=4, t=4.725 t_{s p} ;(\mathrm{d}): u^{\prime} / S_{L}=12, t=3.15 t_{s p} \ldots \ldots \ldots \ldots \ldots \ldots$ 


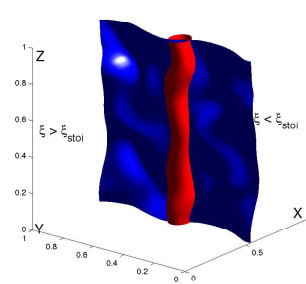

(a) $t=0.0525 t_{s p}$

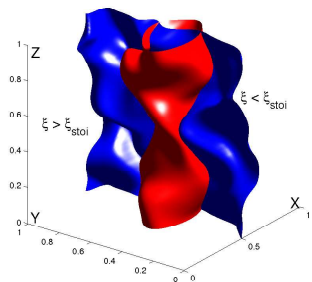

(b) $t=2.1 t_{s p}$

Figure 1: The stoichiometric iso-surface (blue) and the iso-surface of $T=0.5$ (red) for $u^{\prime} / S_{L}=4$.

Single column

Colour figure in printed version 


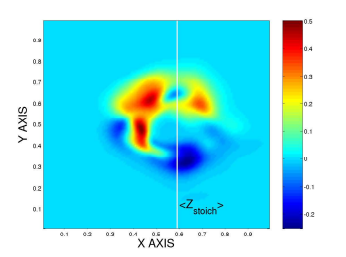

(a) $\left\langle\rho v^{\prime} T^{\prime}\right\rangle$

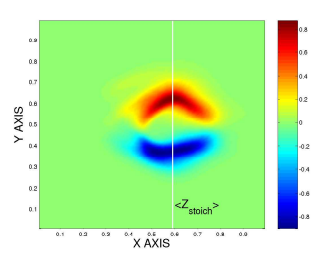

(b) $-D_{t} \partial\langle\rho T\rangle / \partial y$

Figure 2: Contours of unconditional flux in the $y$-direction and of the usual gradient model at $t=2.1 t_{s p}$ and for $u^{\prime} / S_{L}=4$.

Single column

Colour figure in printed version 


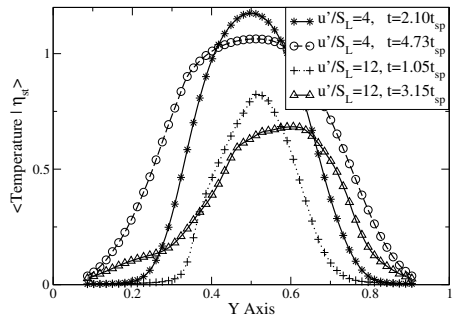

(a) $\left\langle T \mid \eta=\xi_{s t}\right\rangle$

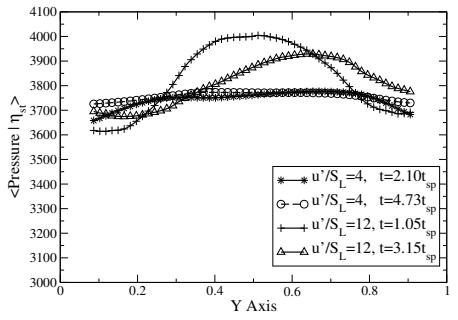

(b) $\left\langle P \mid \eta=\xi_{s t}\right\rangle$

Figure 3: Distributions in the $y$-direction of the mean conditional temperature and pressure at $\eta=\xi_{s t}$ and the indicated times.

Double column 

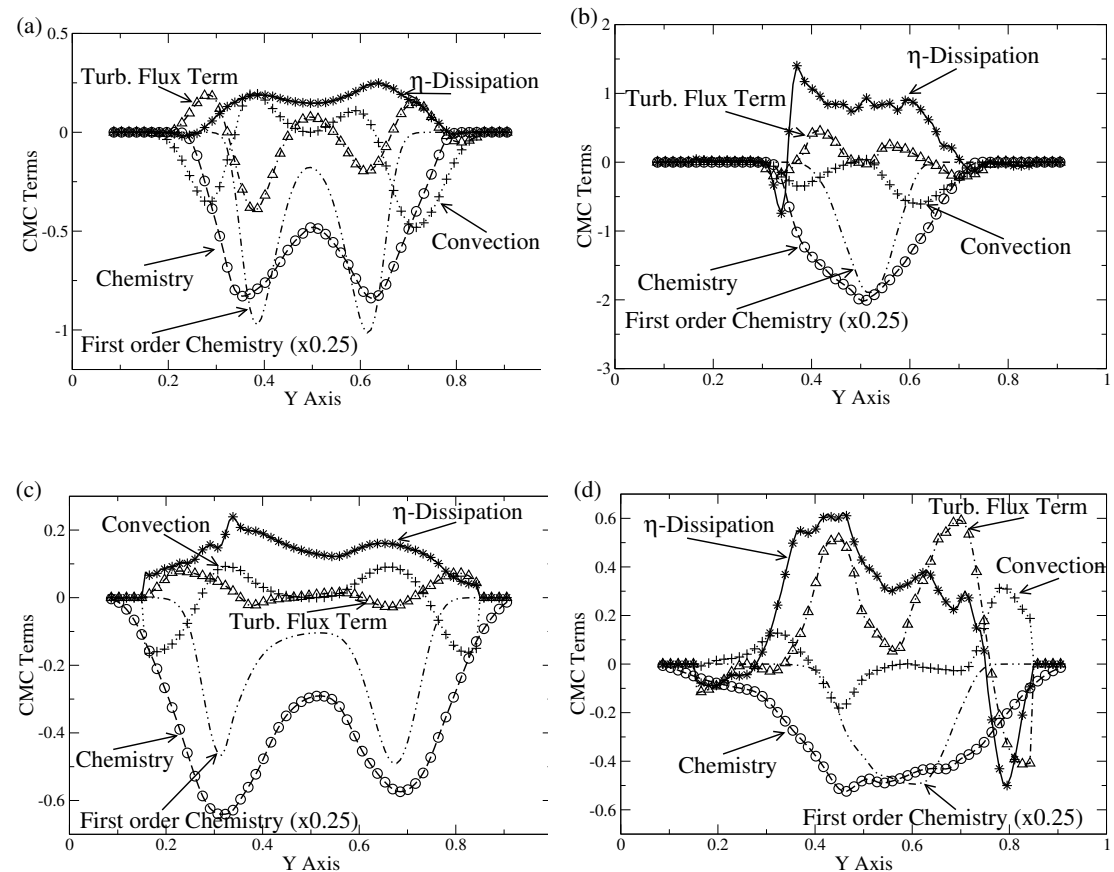

Figure 4: The spatial distribution of the various source terms in the CMC equation at $\eta=\xi_{s t}$. (a): $u^{\prime} / S_{L}=4, t=2.1 t_{s p} ;(\mathrm{b}): u^{\prime} / S_{L}=12, t=1.05 t_{s p} ;(\mathrm{c}): u^{\prime} / S_{L}=4, t=4.725 t_{s p} ;(\mathrm{d}):$ $u^{\prime} / S_{L}=12, t=3.15 t_{s p}$.

\section{Double column}



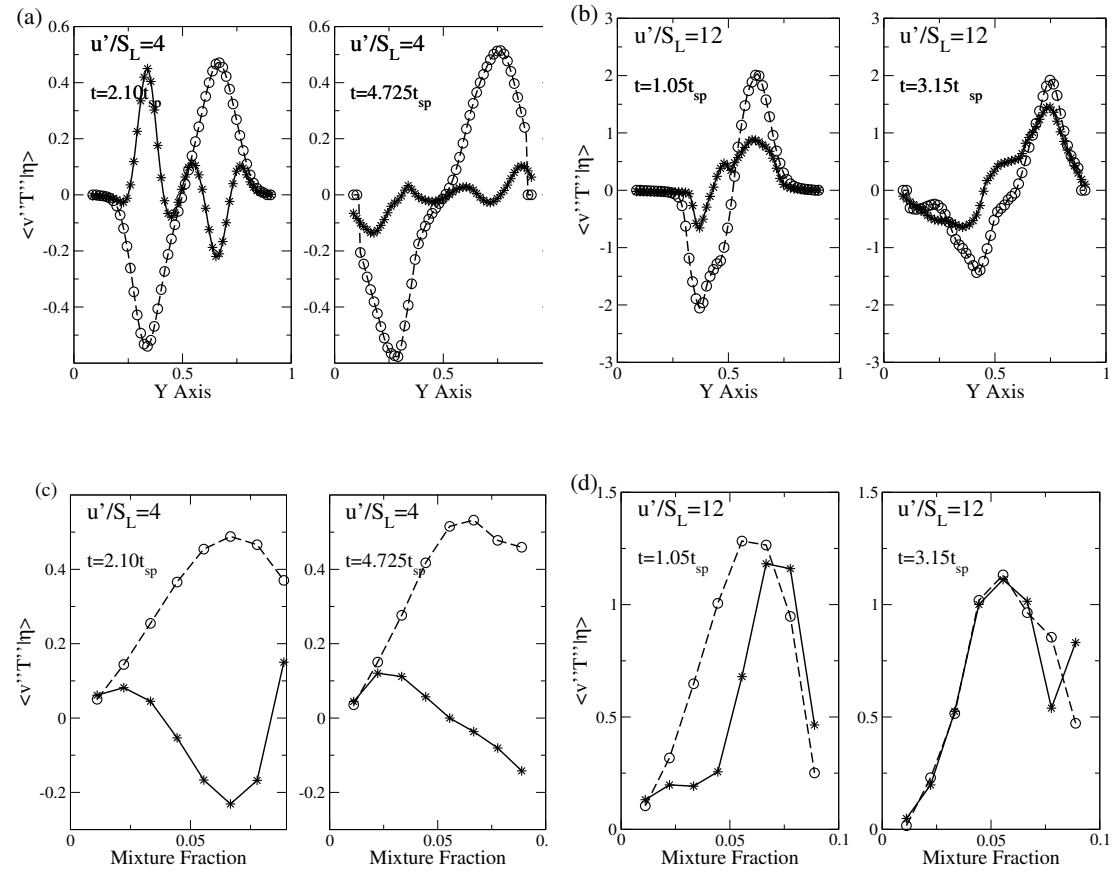

Figure 5: Turbulent flux $\left({ }^{*}\right)$ and gradient diffusion approximation (o) for the indicated $u^{\prime} / S_{L}$ and time. For (a) and (b) the $y$-distribution of $\left\langle v^{\prime \prime} T^{\prime \prime} \mid \eta=\xi_{s t}\right\rangle$ is shown. For (c) and (d), $y=0.5$.

Double column 

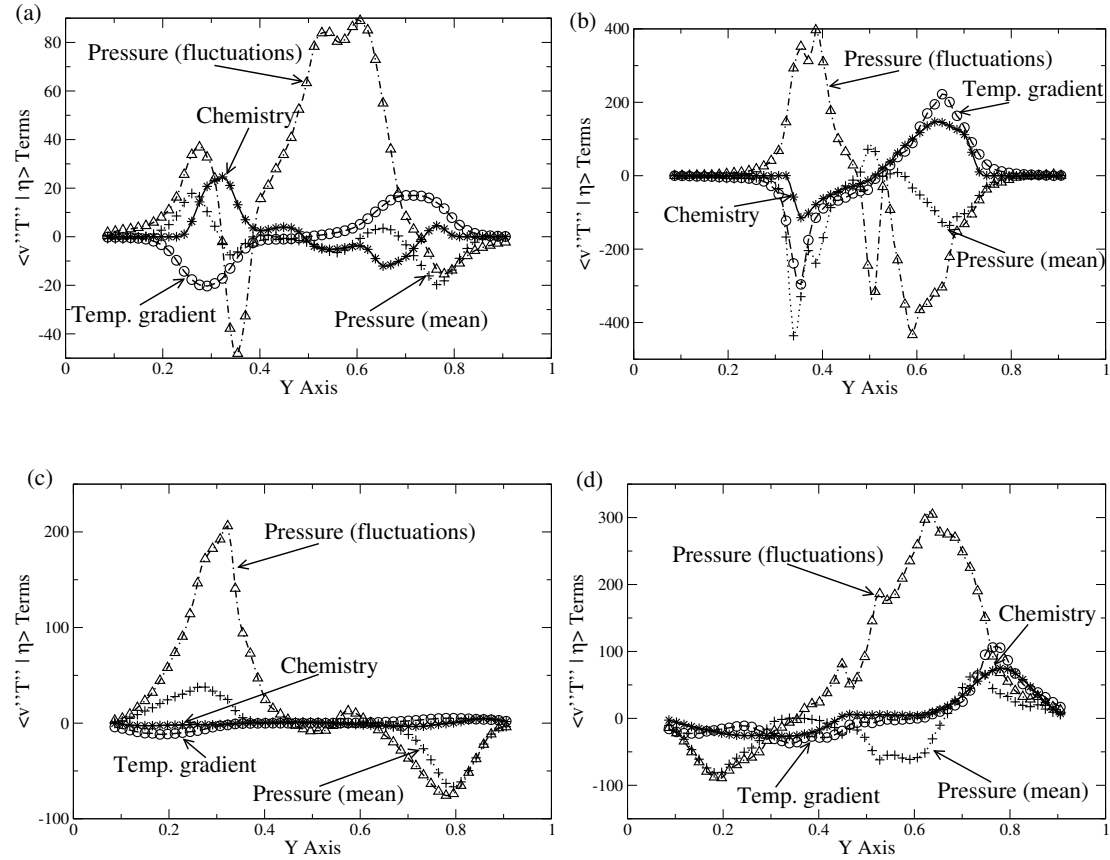

Figure 6: The spatial distribution of the various source terms in the CMC conditional flux equation for $\eta=\xi_{s t}$. (a): $u^{\prime} / S_{L}=4, t=2.1 t_{s p} ;(\mathrm{b}): u^{\prime} / S_{L}=12, t=1.05 t_{s p} ;(\mathrm{c}): u^{\prime} / S_{L}=4, t=4.725 t_{s p}$; (d): $u^{\prime} / S_{L}=12, t=3.15 t_{s p}$

Double column 